(RESEARCH ARTICLE)

\title{
Heart rate variability parameters among acute heart failure patients in a tertiary center in Nigeria
}

\author{
Adeyemi OA ${ }^{1}$, Akinlade OM 1,2, ${ }^{*}$, Ogunmodede JA ${ }^{1}$, Kolo PM ${ }^{1}$, Katibi IA ${ }^{1}$ and Omotoso ABO ${ }^{1}$ \\ ${ }^{1}$ Cardiology unit, Internal Medicine Department, Unilorin Teaching Hospital, Ilorin, Kwara state, Nigeria. \\ ${ }^{2}$ Cardiology unit, Internal Medicine Department, LAUTECH Teaching Hospital, Ogbomoso, Oyo state, Nigeria.
}

Publication history: Received on 23 July 2020; revised on 06 August 2020; accepted on 10 August 2020

Article DOI: https://doi.org/10.30574/wjarr.2020.7.2.0274

\begin{abstract}
The morbidity and mortality associated with heart failure remain high despite advancement in its management hence underscoring the need for further detection of factors contributing to poor outcome. Heart rate variability (HRV) has been shown to predict outcomes in various cardiovascular disease and sudden cardiac death (SCD). This study therefore, was designed to define the relationship between 24-hour Holter electrocardiographic parameters at admission and the outcome of hospital admission among acute heart failure patients.

The study was a hospital-based prospective cohort study. One hundred and fifty subjects with acute heart failure and same control subjects were recruited. All participants had clinical evaluation with 24 hours Holter ECG and Echocardiographic studies. Recruited subjects were closely monitored throughout the hospital admission period for clinical events. The outcomes of hospital admission were determined and documented accordingly.

One hundred and forty cases and one hundred and forty controls were eventually analyzed. The mean ages of both cases and controls were similar, likewise their sex distribution and other anthropometric parameters. Heart rate variability analysis showed that proportion of R-R interval that differs by more than $50 \mathrm{msec}$ (pNN50) was significantly lower among in-hospital deaths compared to survivors. Also, at 30-days post discharge, both pNN50 and standard deviation of normal to normal R-R intervals (SDNN) were significantly lower among patients that died than in those that survived. There was weak positive correlation between Heart rate variability and myocardial systolic functions, while there was stronger negative correlation with diastolic functions.
\end{abstract}

Keywords: Cardiovascular disease; Heart failure; Heart rate variability; Sudden cardiac death

\section{Introduction}

Heart failure (HF) is a clinical condition associated with poor outcome and has a rising prevalence in sub-Saharan Africa and globally. It is a growing public health problem affecting about 23 million people worldwide especially in individuals above 45 years therefore, it is regarded as the epidemic of the 21 st century $(1,2)$. Acute Heart Failure (AHF) is the term used to describe the rapid onset of, or change in symptoms and signs of HF. It is a life-threatening condition that requires immediate medical attention and usually leads to urgent admission to the hospital (3-5).

Risk stratification and early detection of various factors contributing to cardiovascular morbidity and mortality thus becomes very important. Studies have shown that heart rate variability (HRV) and other repolarization dynamics and variability could predict outcomes in various cardiovascular disease $(4,6)$. For instance, reduced HRV has been shown to be associated with worse prognosis. Nikolaos et al reported that low HRV following acute coronary events of myocardial ischaemia/infarction is associated with high incidence of SCD and this has been related to increased risk of

\footnotetext{
${ }^{*}$ Corresponding author: Akinlade OM

Cardiology unit, Internal Medicine Department, Unilorin Teaching Hospital, Ilorin, Kwara state, Nigeria
} 
fatal ventricular arrhythmias (7). Lasisi et al, also showed that ambulant out-patients with chronic HF have significantly narrow HRV compared to age and sex matched controls (8). Some studies conducted among cardiac disease patients suggest that decreased HRV as well as baroreceptor reflex dysfunction are more powerful predictors for cardiovascular mortality than established clinical predictors, such as LVEF and ventricular premature contractions (PVCs) $(6,9)$. HRV is significantly reduced in patients with LVH secondary to hypertension or aortic valve diseases (10).

The relationship between HRV parameters and indices of left ventricular function as well as their correlation with mortality from HF has been well documented in studies among Caucasian population but not so well for the Nigerian subjects. The few reported studies in Nigeria focused on ambulant outpatient CHF patients. There is dearth of information in this regard for admitted AHF patients and specifically from our own community, particularly in the North-central Nigeria. This study therefore aims to determine the relationship between 24- hour ECG indices (with emphasis on HRV) and both outcome of hospital admission and 30-day mortality among AHF patients.

\section{Methodology}

The research work is a hospital based prospective cohort study conducted at the University of Ilorin Teaching Hospital (UITH) Ilorin, Kwara State.

\subsection{Equipment}

Schiller $₫$ MT 101 Holter ECG recording system, Sonoscape $®$ SSI-8000 Echocardiogram machine, Stadiometer, secca ${ }^{\circledR}$ bathroom weighing scale, mercury sphygmomanometer, non-elastic tape measure, computer, Events diary.

\subsection{Ethical Consideration}

Approval from the Ethics and Research Committee of UITH was sought and obtained; the cost for the study was borne by the researcher. The researcher was financially supported by a dissertation allowance from UITH Ilorin.

\subsubsection{Study Subjects}

All patients admitted via the Emergency unit and Cardiology outpatient clinic of Department of Medicine of UITH, presenting with AHF were consecutively recruited as they presented based on inclusion criteria for subjects.

\subsubsection{Study control subjects}

Age and sex matched control participants were recruited, mainly from members of staff in UITH, willing healthy participants from the community and tertiary school students.

\subsubsection{Inclusion Criteria for patients}

- $\quad$ AHF patients who are 18 years and above.

- Decompensation of previously diagnosed HF.

- $\quad$ Newly diagnosed AHF patients with onset of symptoms not greater than three weeks.

- $\quad$ Patients diagnosed with HF and commenced on standard therapy for HF including diuretics, aldosterone antagonists and angiotensin-converting enzyme inhibitors (ACEIs)/angiotensin receptor blockers (ARBs) for a period not more than 24 to 48 hours.

\subsubsection{Exclusion criteria for patients}

- $\quad$ Patients whose symptoms could be explained by any other co-morbidities e.g. chronic kidney disease, chronic liver disease. etc.

- Poor echocardiographic window resulting from conditions such as chronic obstructive pulmonary disease (COPD) and Obesity.

- $\quad$ HF patients who died within the first 24 hours of admission before Holter recording could be completed.

\subsection{Subjects Selection and method of sampling}

- $\quad$ All patients admitted for AHF who fulfilled the inclusion criteria from both cardiology outpatient clinics and emergency department were recruited consecutively into the study.

- Informed consent; Informed written consent was obtained from the prospective participants using a consent form which stated in clear and simple terms the purpose and importance of the study including the involved procedures. 
- $\quad$ Data Collection

- Demographic and relevant clinical information was obtain from the patients or care-givers and fed into a structured questionnaire.

\subsubsection{Protocol 1}

Establishing Clinical Heart Failure

- $\quad$ Clinical evaluation for the diagnosis of AHF were performed by the investigator for all recruited subjects based on the Framingham's criteria of the concurrent presence of two major criteria or one major with two minor criteria (11).

\subsubsection{Protocol 2}

Anthropometric measurements

- $\quad$ Anthropometric measurements at recruitment such as weight $(\mathrm{kg})$, height $(\mathrm{m})$ and waist circumference (cm) were taken. The height of subjects was measured to the nearest $0.1 \mathrm{~m}$ using a stadiometer with the subject in erect position without foot wear or headgear. The weight of the patients shall be measured with light clothing on using a secca scale to the nearest $0.1 \mathrm{~kg}$.

- A thorough physical examination with emphasis on the cardiovascular system was performed by the investigator.

\subsubsection{Protocol 3}

\section{Echocardiography}

- $\quad$ Conventional trans-thoracic echocardiography (TTE) was used to evaluate the subjects using Sonoscapeß SSI8000 Echocardiogram machine, 2013 model. Two-dimensional (2-D) Echo was obtained using conventional positions and views. For 2D directed M mode measurement; parasternal long axis (PLAX) view was used. Measurements were made in line with the American Society of Echocardiography (ASE) convention (12).

- $\quad$ Three measurements were taken for each parameter and the average taken for recording on the proforma. Specific M mode measurements were appropriately taken at end-diastole and end-systole using concurrent electrocardiogram for timing the cardiac cycles.

- $\quad$ Echocardiographic Left Ventricular hypertrophy (LVH) was defined by ASE guideline of $>95 \mathrm{~g} / \mathrm{m} 2 \mathrm{in} \mathrm{female}$ and $115 \mathrm{~g} / \mathrm{m} 2$ in males respectively (12). Doppler echocardiography was obtained according to the recommendations of quantification of Doppler Echocardiography of the ASE (13). The LV diastolic flow pattern was assessed by Doppler studies via the Apical 4 chamber and 5 chamber views. The trans-mitral, pulmonary and aortic velocities were determined.

\subsubsection{Protocol 4}

Holter studies

A 2-channel Holter monitor was attached to the patient's chest within the first 24 hours of patient's admission into the hospital. Adequate skin preparation was carried out before the leads were attached and secured with skin adhesive plasters.

Reading and analysis of the recordings

The monitor was detached after the completion of the study period of 24 hours and analysis was done by the computer software provided by Schiller MT 200® Holter analysis software. Visual editing and re-classification of the entire recording was carried out by the researcher.

HRV parameters that were used include; pNN50 (proportion of N-N interval greater than 50msec), SDNN (standard deviation of normal to normal intervals) and SDANN (standard deviation of average normal to normal intervals) $(6,9,10,14)$. Various forms of arrhythmias as detected by the monitor and observed during editing and assessment were noted. The relationship between these parameters and the outcome of the hospital admission and 30-day post discharge mortality as well as the echocardiographic findings of the patients were determined. 


\subsubsection{Protocol 5}

Admission, discharge and subjects' follow-up

The patients were treated according to the international guidelines on AHF management as adopted by the Cardiology unit of the Department of Medicine, UITH. All recruited patients were followed up accordingly, while on admission and up to 30 days after discharge. The end point of the study was death of patients while on admission or 30-days post discharge from the hospital.

\subsection{Data Analysis}

All the Holter ECG parameters and echocardiographic left ventricular indices obtained were input into the computer and analyzed using the Statistical Package for the Social Sciences (SPSS) version 23.0 for windows. Measures of central tendency and dispersion of quantitative variables was determined. Chi Square test and student t test were used to test the significance of the differences between proportions of categorical variables and means of continuous variables respectively. The confidence level was set at $95 \%$ and level of significance at $\mathrm{P}<0.05$.

Mann-Whitney U test was used to test the differences between Holter parameters of patients and controls and also to determine the differences between mortality and survivors among AHF subjects. The Spearman's rank correlation test was used to determine the correlation between HRV and ventricular functions as well as cardiac arrhythmias and ventricular function. Linear regression analysis model was used to determine independent risk factors predictive of patient's outcome.

\section{Results}

A total of 300 subjects (150 cases and 150 age and sex-matched controls) were recruited into the study. However, 10 results were invalidated among the cases due to presence of greater than 10 of total QRS counts that were categorized as artifacts. Therefore, 140 AHF cases and 140 controls were analyzed. The mean age of the AHF cases was 53.06 $\pm 16.07 \mathrm{yrs}$, while the mean age for the control group was $52.55 \pm 14.50 \mathrm{yrs}$. The AHF group comprised $72 \mathrm{males}(51.40 \%)$ and 68 females (48.60\%) while the control group consisted of 74 males (52.90\%) and 66 females (47.10\%).

\subsection{Heart rate and HRV parameters of AHF subjects and controls}

Our study showed that ambulatory heart rate parameters were significantly higher among AHF patients compared with the control group. The median value of the mean heart rate for AHF patients was 88.0 (78.0 - 96.0 beats/min) while that of the control group was 70.0 (69.0 - 74.0 beats $/ \mathrm{min}),(\mathrm{p}<0.001)$. Tachycardia episodes were more frequent among AHF cases, with median value of 67.0 (25.5 - 118.0) compared with $40.0(6.0-60.0)$ for the control group, ( $p<0.001)$.

Table 1 Heart rate and HRV parameters of AHF subjects and controls

\begin{tabular}{|l|l|l|l|}
\hline & Patients & Control & $\boldsymbol{p}$ value \\
\hline Variable & Median (IQR) & Median (IQR) & \\
\hline Mean heart rate & $88.0(78.0-96.0)$ & $70.0(69.0-74.0)$ & $<0.001^{*}$ \\
\hline Minimum heart rate & $54.0(39.0-77.0)$ & $44.0(42.0-44.0)$ & $<0.001^{*}$ \\
\hline Maximum heart rate & $160.0(146.0-200.8)$ & $126.0(110.0-145.0)$ & $<0.001^{*}$ \\
\hline AHRR & $110.0(76.0-135.0)$ & $82.0(64.0-103.0)$ & $<0.001^{*}$ \\
\hline $\begin{array}{l}\text { Tachycardia episodes in } 24 \\
\text { hours }\end{array}$ & $67.0(25.5-118.0)$ & $40.0(6.0-60.0)$ & $<0.001^{*}$ \\
\hline HRV & & & \\
\hline pNN50 & $10.1(7.2-25.0)$ & $19.5(9.4-20.6)$ & $<0.001^{*}$ \\
\hline SDANN & $31.0(25.0-41.4)$ & $89.0(79.0-92.0)$ & $<0.001^{*}$ \\
\hline SDNN & $73.00(45.25-82.5)$ & $117.0(105.0-121.0)$ & $<0.001^{*}$ \\
\hline
\end{tabular}

AHRR: Ambulatory heart rate range, IQR: interquartile range, HRV: Heart rate variability, PNN50: Proportion of normal R-R interval greater than $50 \mathrm{msec}$, SDANN: average standard deviation of normal R-R intervals, SDNN: standard deviation of normal to normal R-R intervals, *: p value <0.05. 
Median HRV indices (PNN50, SDNN, SDANN) were lower in AHF group compared to the control ( $p=0.000,0.0000$ and 0.00 respectively). The differences in values were all statistically significant as shown in table 1.

\subsection{Heart rate variability and left ventricular functions}

The study did not find significant correlation between left ventricular systolic function as assessed by EF and parameters of heart rate variability. However, total pNN50 and all SDANN indices showed correlation with LV myocardial tissue doppler systolic velocity ( $\left.s^{\prime}\right)$. While SDANN tend to reduce in value with increase in myocardial tissue doppler systolic velocity ( $\left.\mathrm{s}^{\prime}\right)$, pNN50 increases with increase in myocardial tissue doppler systolic velocity ( $\left.\mathrm{s}^{\prime}\right)$. This relationship was statistically significant for both SDANN ( $\mathrm{p}=0-005)$ and pNN50, $(\mathrm{p}=0.017)$ as shown in table 2 .

Table 2 Relationship between HRV and Left ventricular systolic functions

\begin{tabular}{|l|l|l|l|l|}
\hline & \multicolumn{2}{|l|}{ Ejection fraction } & S average & \multicolumn{2}{l|}{} \\
\hline HRV & r & $\boldsymbol{p}$ value & r & $\boldsymbol{p}$ value \\
\hline pNN50 day & 0.138 & 0.105 & 0.182 & $0.032^{*}$ \\
\hline pNN50 night & -0.022 & 0.799 & 0.236 & $0.005^{*}$ \\
\hline pNN50 all & 0.056 & 0.512 & 0.202 & $0.017^{*}$ \\
\hline SDANN day & -0.035 & 0.685 & 0.222 & $0.008^{*}$ \\
\hline SDANN night & 0.018 & 0.832 & 0.291 & $<0.001^{*}$ \\
\hline SDANN all & -0.022 & 0.798 & 0.236 & $0.005^{*}$ \\
\hline SDNN day & 0.099 & 0.245 & -0.072 & 0.401 \\
\hline SDNN night & -0.082 & 0.334 & -0.139 & 0.101 \\
\hline SDNN all & -0.009 & 0.920 & -0.120 & 0.159 \\
\hline
\end{tabular}

HRV: Heart rate variability, PNN50: Proportion of normal R-R interval greater than 50msec, SDANN: average standard deviation of normal R-R intervals, SDNN: standard deviation of normal to normal R-R intervals, IQR: interquartile range, r: Spearman Correlation Coefficient, ${ }^{*} p$ value $<0.05$ = statistical significance.

The left ventricular filling E/A ratio had a weak negative correlation with pNN50. As left ventricular E/A ratio increase, pNN50 reduces. The SDANN however has a stronger positive relationship with mitral E/A such that as the SDANN increases, the mitral E/A increases in value towards restrictive pattern of ventricular filling (Table 3).

Table 3 Diastolic function and Heart Rate Variability

\begin{tabular}{|l|l|l|l|l|}
\hline & \multicolumn{2}{|l|}{ E/A average } & E/e' average \\
\hline HRV & $\mathbf{r}$ & $\boldsymbol{p}$ value & $\mathbf{r}$ & $\boldsymbol{p}$ value \\
\hline pNN50 day & -0.128 & 0.131 & -0.165 & 0.051 \\
\hline pNN50 night & -0.259 & $0.002^{*}$ & -0.137 & 0.107 \\
\hline pNN50 all & -0.197 & $0.020^{*}$ & -0.139 & 0.102 \\
\hline SDANN day & 0.316 & $<0.001^{*}$ & 0.034 & 0.686 \\
\hline SDANN night & 0.299 & $<0.001^{*}$ & 0.021 & 0.802 \\
\hline SDANN all & 0.302 & $<0.001^{*}$ & 0.000 & 0.997 \\
\hline SDNN day & 0.109 & 0.201 & 0.152 & 0.074 \\
\hline SDNN night & 0.128 & 0.132 & 0.156 & 0.066 \\
\hline SDNN all & 0.158 & 0.062 & 0.082 & 0.334 \\
\hline
\end{tabular}

HRV: Heart rate variability, pNN50: Proportion of normal R-R interval greater than 50msec, SDANN: average standard deviation of normal R-R intervals, SDNN: standard deviation of normal to normal R-R intervals, r: Spearman Correlation Coefficient, ${ }^{*} p$ value $<0.05=$ statistical significance 


\subsection{HRV parameter and outcome of hospital Admission/ 30-day post discharge mortality from AHF}

There was no statistically significant difference between the HR parameters of those that survived and those that died during hospital admission. However, among the HRV parameters, pNN50 showed statistically significant difference between the survivors and the dead patients while on admission. pNN50 was significantly lower among AHF nonsurvivors $6.30(1.10-10.30 \mathrm{msec})$ compared to survivors $10.90(7.70-30.10 \mathrm{msec}), \mathrm{p}=0.007$ (Table 4).

Table 4 Relationship between Heart Rate and HRV parameter with outcome of hospital Admission

\begin{tabular}{|l|l|l|l|}
\hline & Discharged & Deaths & $\boldsymbol{p}$ value \\
\hline Variable & Median (IQR) & Median (IQR) & \\
\hline Mean heart rate & $88.0(78.0-96.0)$ & $92.0(92.0-108.0)$ & 0.064 \\
\hline Minimum heart rate & $61.0(48.0-80.0)$ & $67.0(57.0-94.0)$ & 0.313 \\
\hline Maximum heart rate & $163.0(150.0-200.0)$ & $170.0(132.0-200.0)$ & 0.803 \\
\hline AHRR & $115.0(83.5-147.0)$ & $86.0(69.0-168.0)$ & 0.771 \\
\hline $\begin{array}{l}\text { Tachycardia episodes in } \\
\text { 24 hours }\end{array}$ & $74.0(43.0-116.0)$ & $81.0(21.0-118.0)$ & 0.543 \\
\hline HRV & & & \\
\hline pNN50 & $10.9(7.7-30.1)$ & $6.3(1.1-10.3)$ & $0.007^{*}$ \\
\hline SDANN & $31.0(25.0-41.4)$ & $26.0(8.0-95.0)$ & 0.622 \\
\hline SDNN & $72.0(45.5-82.0)$ & $90.0(12.0-117.0)$ & 0.074 \\
\hline
\end{tabular}

AHRR; Ambulatory Heart rate range, HRV: Heart rate variability, pNN50: Proportion of normal R-R interval greater than 50msec, SDANN: average standard deviation of normal R-R intervals, SDNN: standard deviation of normal to normal R-R intervals, *: p value $<0.05$

There was no statistically significant difference in heart rate parameters between survivors after 30 days following hospital discharge and those that died from HF related illness within 30 days of discharge. However, HRV parameters of PNN50 and SDNN were significantly lower among AHF patients that died within 30 days of discharge from the hospital compared to those that survived (Table 5).

Table 5 Heart rate parameters and 30-day mortality

\begin{tabular}{|l|l|l|l|}
\hline & Survivors & Deaths & $\boldsymbol{p}$ value \\
\hline Variable & Median (IQR) & Median (IQR) & \\
\hline Mean heart rate & $88.0(76.0-96.0)$ & $89.0(88.0-100.0)$ & 0.065 \\
\hline Minimum heart rate & $57.5(48.0-77.0)$ & $74.0(57.5-81.0)$ & 0.075 \\
\hline Maximum heart rate & $168.0(150.0-197.0)$ & $160.0(145.0-200.0)$ & 0.814 \\
\hline AHRR & $113.0(87.0-141.3)$ & $120.0(76.0-147.0)$ & 0.869 \\
\hline $\begin{array}{l}\text { Tachycardia episodes in } \\
\text { 24hours }\end{array}$ & $74.0(46.0-120.0)$ & $85.0(43.0-116.0)$ & 0.727 \\
\hline HRV & & & \\
\hline pNN50 & $12.0(7.7-38.0)$ & $8.7(7.4-12.4)$ & $0.026^{*}$ \\
\hline SDANN & $32.5(18.0-43.0)$ & $27.0(26.0-31.0)$ & 0.179 \\
\hline SDNN & $73.0(55.8-82.5)$ & $52.0(43.0-75.50)$ & $0.045^{*}$ \\
\hline
\end{tabular}

AHRR; Ambulatory Heart rate range; U: Mann Whitney U test; * $\mathrm{p}$ value $<0.05$ 


\section{Discussion}

\subsection{Heart rate variability among AHF patients and the control group}

This study showed that AHF subjects were found to have lower pNN50, SDNN and SDANN compared to controls. These were consistent with findings from previous study in the Southwest Nigeria reported that HRV parameters (SDNN, pNN50 and RMSSD) were significantly lower among chronic ambulant HF patients compared with the controls (8). Likewise studies done among Caucasians chronic HF patients revealed similar findings of lower HRV among HF subjects compared with controls (16-19). Heart rate and its variability are under sympatho-vagal influence, therefore reduced $\mathrm{HRV}$ and increased heart rate are thought to be as a result of autonomic imbalance with reduced vagal tone and increased sympathetic activity $(14,15,19)$.

Among time-domain measures of HRV, SDNN and SDANN reflect both sympathetic and parasympathetic modulation of cardiac activities with reduced SDANN and SDNN values usually indicating relative sympathetic dominance. pNN50, on the other hand is a direct marker of parasympathetic activity $(20,21)$. Reduced pNN50 as found in this study is a marker of reduced parasympathetic tone and increased sympathetic tone leading to sympathetic dominance which has been shown to be proarrhythmic in other studies (22-24).

\subsection{Heart rate variability and ventricular functions}

The ventricular functions have systolic and diastolic components, which are interrelated. While systolic function is directly related to the myocyte contractile function, diastolic function reflects the myocardial elasticity and stiffness which is more related to the intercellular matrix. The relationship between these functions and HRV was assessed in this study. While pNN50 showed weak positive correlation with (s'), SDANN however showed weak negative correlation with myocardial systolic motion (s') There was no statistically significant relationship between left ventricular EF and HRV. This finding was however different from the findings by Hoffmann et al while assessing baroreceptor sensitivity, HRV and systolic function among idiopathic dilated cardiomyopathy patients (25). They found that there was a weak correlation between left ventricular EF and SDNN. Likewise, in a study done by Joshi et al, there was a weak correlation between LVEF and HRV among patients at high risk for CVDs (26). However, Birand et al found a strong correlation between frequency domain analysis of HRV and Doppler echocardiographic indices of left ventricular systolic performance (s') in patients with CAD (27). Reportage of this relationship among HF patient is however scanty. The difference in the findings in this studies may be ascribed to the difference in the cohort group used for the studies.

On the other hand, diastolic function showed more a consistently negative correlation with the parameters of HRV. Therefore, it can be inferred from the result of this study that as the diastolic E/A increases, ventricular filling pressure increases, HRV reduces.

\subsection{Heart rate, Variability and outcome}

This study showed that Heart rate parameters between the AHF patients that died on admission and those that survived were statistically similar. The mean heart rate for both survivors and non-survivors was below 100 beats $/ \mathrm{min}$. However, the normality range for mean Heart rate in Holter is yet to be defined but some studies have shown that there is a linear relationship between heart rate and mortality. There is an increase in mortality with rise in mean heart rate above 60 beats/min $(28,29)$.

The study also showed that HRV parameters were lower among those that died from HF during admission as well as within 30 days of discharge. For in-hospital mortality, pNN50 was significantly lower among those that died during admission compared to those that were discharged. Both SDNN and pNN50 were significantly lower among those patients that died within 30 days of discharge than the survivors beyond 30 days.

Therefore, in this study, Low pNN50 was associated with both in-hospital mortality and 30-day mortality whereas, low SDNN was associated with only 30 -day mortality. Ponikowski et al in a study done about 2 decades ago amongst patients with moderate to severe HF similarly found lower HRV in patients who died compared to the controls and likewise Low SDNN was associated with mortality in their study (30). Also, Nolan et al reported that SDNN was the most powerful predictor of risk of death from progressive pump failure among stable CHF patients (31). 


\section{Conclusion}

\section{Recommendations}

- Complementary risk analysis using Holter ECG can provide additional information among AHF patients and therefore should be undertaken as part of further work up among AHF patients.

- Heart rate variability can be used to identify patients at high risk for mortality during admission and in the immediate post-discharge period. More intensive monitoring can then be offered to such patients.

- There is need for Larger randomized control trials on HRV and mortality to further strengthen the evidence provided by this study.

\section{Study limitations}

- $\quad$ Limited period was available for follow-up of the patients thereby limiting the long term predictive power of the study.

- $\quad$ Accurate determination of sudden death could not be undertaken as there was no continuous ECG monitoring after admissions on the wards where the study was undertaken.

Cultural practices in our locality do not encourage patients to be brought into hospital in order to conduct a post-mortem study amongst patients after death either at home or even in the hospital.

\section{Compliance with ethical standards}

\section{Acknowledgments}

We acknowledge resident doctors in UITH for assistance during the project.

\section{Disclosure of conflict of interest}

No conflicts of interest.

\section{References}

[1] Khatibzadeh S, Farzadfar F, Oliver J, Ezzati M and Moran A. (2012). Worldwide risk factors for heart failure. A systematic review and pooled data analysis. Int J Cardiol, 168(2), 1-9.

[2] Cygankiewicz I, Zaręba W and Bayes de Luna A. (2008). Prognostic value of Holter monitoring in congestive heart failure. Cardiol J, 15(4), 313-323.

[3] Clyde WY, Mariell J, Biykem B, Javed B, Donald EC Jr, Mark H, et al. (2013). ACCF/AHA Guideline for the Management of Heart Failure: A Report of the American Guidelines College of Cardiology Foundation/American Heart Association Task Force on Practice Guideline. Circulation, 15(24), 4539.

[4] John JV, Stamatis A, Stefan DA, Angelo A, Michael B, Kenneth D, et al. (2012). ESC Guidelines for diagnosis and treatment of Acute and Chronic Heart Failure. Eur Heart J, 33(10), 1787-1847.

[5] Damasceno A, Mayosi BM, Sani M, Ogah OS, Charles M, Ojji D, et al. (2012). The causes, treatment, and outcome of Acute Heart Failure in 1006 Africans from 9 Countries (THESUS-HF) Ach Intern Med, 172(18), 1386-1394.

[6] Nikolaos D and Hindricks G. (2013). Risk Stratification after Myocardial Infarction. Eur Heart J, 34(26), 19641971.

[7] Heart Rate Variability: Standards of Measurement, Physiological Interpretation, and Clinical Use. Task Force of the European Society of Cardiology the North American Society of Pacing Electrophysiology. Eur Heart J. (1996).17, 354-381.

[8] Lasisi GT, Adebola AP, Ogah OS and Daniel FA. (2012). Prevalence of Ventricular arrhythmias and heart rate variability pattern in chronic heart failure. Niger Postgrad Med J, 19(3), 157-162.

[9] Crawford MH, Bernstein SJ, Deedwania PC, DiMarco JP, Ferrick KJ, Garson A, et al. (1999). ACC/AHA guidelines for ambulatory electrocardiography: a report of the American College of Cardiology/American Heart Association Task Force on Practice Guidelines developed in collaboration with the North American Society for Pacing and Electrophysiology. J Am Coll Cardiol, 34(3), 912-948. 
[10] Knoebel SB, Crawford MH, Dunn M, Fisch C, Forrester J, Hutter A, et al. (1989). Guidelines for ambulatory electrocardiography. A report of the American College of Cardiology/American Heart Association Task Force on Assessment of Diagnostic and Therapeutic Cardiovascular Procedures (Subcommittee on Ambulatory Electrocardiography). Circulation, 79(1), 206-215.

[11] Vasan RS, Benjamin EF, Larson MG, Leip EP, Wang TJ, Wilson PW, et al. (2002). Plasma natriuretic Peptides for community screening for left ventricular hypertrophy and systolic dysfunction. The Framingham heart study. JAMA, 288(10), 1252-1259.

[12] Lang RM, Badano LP, Mor-Avi V, Afilalo J, Armstrong A, Ernande L, et al. (2015). Recommendations for cardiac chamber quantification by echocardiography in adults: an update from the American Society of Echocardiography and the European Association of Cardiovascular Imaging. J Am Soc Echocardiogr, 28(1), 1-39.

[13] Quinines MA, Otto MC, Stoddard M, Waggoner A and Zoghbi WA. (2002). Recommendations for the quantification of Doppler echocardiography: A report from the Doppler quantification task force of the Nomenclature and Standard Committee of the American Society of Echocardiography. J Am Soc Echocardiogr, 15, 167-184.

[14] Camm. A and Malik M. (1990). Heart Rate Variability. Electrophysiology, Pacing and Arrhythmia. J of Clin Cardiol, $13,570-576$.

[15] Sliwa K, Davison BA, Mayosi BM, Damasceno A, Sani M, Ogah OS, et al. (2013). Readmission and death after an acute heart failure event: predictors and outcomes in sub-Saharan Africa: results from the THESUS-HF registry. Eur Heart J, 34(40), 3151-3159.

[16] Ponikowski P, Anker SD, Chua TP, Szelemej R, Piepoli M, Adamopoulos S, et al. (1997). Depressed heart rate variability as an independent predictor of death in chronic congestive heart failure secondary to ischemic or idiopathic dilated cardiomyopathy. Am J Cardiol, 79(12), 1645-50.

[17] Galinier M, Pathak A, Fourcade J, Androdias C, Curnier D, Varnous S, et al. (2000). Depressed low frequency power of heart rate variability as an independent predictor of sudden death in chronic heart failure. Eur Heart J, 21(6), 475-82.

[18] Ho KH, Kalon KL, Moody GB, Peng C, Mietus JE, Larson MG, et al. (1994). Predicting Survival in Heart Failure Case and Control Subjects by Use of Fully Automated Methods for Deriving Nonlinear and Conventional Indices of Heart Rate Dynamics. Circulation, 96(3), 842-848.

[19] Stein PK, Bosner MS, Kleiger RE and Conger BM. (1994). Heart rate variability: a measure of cardiac autonomic tone. Am Heart J, 127(5), 1376-81.

[20] Lombardi F. (2002). Clinical implications of present physiological understanding of HRV components. Cardiac electrophysiol Rev, 6(3), 245-9.

[21] Stein PPK and Kleiger MRE. (1999). Insights from the study of heart rate variability. Annu rev-med, 50(1), 24961.

[22] Tsuji H, Larson MG, Venditti FJ, Manders ES, Evans JC, Feldman CL, et al. (1996). Impact of reduced heart rate variability on risk for cardiac events The Framingham Heart Study. Circulation, 94(11), 2850-5.

[23] Bilchick KC, Fetics B, Djoukeng R, Fisher SG, Fletcher RD, Singh SN, et al. (2002). Prognostic value of heart rate variability in chronic congestive heart failure (Veterans Affairs' Survival Trial of Antiarrhythmic Therapy in Congestive Heart Failure). Am J Cardiol, 90(1), 24-8.

[24] Brouwer J, van Veldhuisen DJ, In't Veld AJM, Haaksma J, Dijk WA, Visser KR, et al. (1996). Prognostic value of heart rate variability during long-term follow-up in patients with mild to moderate heart failure. J Am Coll Cardiol, 28(5), 1183-9.

[25] Hoffmann J, Grimm W, Menz V, Müller H and Maisch B. (2000). Heart rate variability and baroreflex sensitivity in idiopathic dilated cardiomyopathy. Heart, 83(5), 531-8.

[26] Joshi M, Desai K and Menon M. (2015). Correlation between Heart Rate Variability and Left Ventricular Ejection Fraction (LVEF) for Diabetics and Diabetics with Hypertension. Br J Med Res, 2(1), 63-76.

[27] Birand A, Kudaiberdieva GZ, Batyraliev TA, Akgül F and Saliu S. (1998). Relationship between components of heart rate variability and Doppler echocardiographic indices of left ventricular systolic performance in patients with coronary artery disease. Int J Angiol, 7(3), 244-8.

[28] Knoebel SB, Crawford MH, Dunn M, Fisch C, Forrester J, Hutter A, et al. (1989). Guidelines for ambulatory electrocardiography. A report of the American College of Cardiology/American Heart Association Task Force on 
Assessment of Diagnostic and Therapeutic Cardiovascular Procedures (Subcommittee on Ambulatory Electrocardiography). Circulation, 79(1), 206-15.

[29] Fox K, Borer JS, Camm AJ, Danchin N, Ferrari R, Sendon JL, et al. (2007). Resting heart rate in cardiovascular disease. J Am Coll Cardiol, 50(9), 823-30.

[30] Ponikowski P, Anker SD, Amadi A, Chua TP, Cerquetani E, Ondusova D, et al. (1996). Heart rhythms, ventricular arrhythmias, and death in chronic heart failure. J Card Fail, 2(3), 177-83.

[31] Nolan J, Batin PD, Andrews R, Lindsay SJ, Brooksby P, Mullen M, et al. (1998). Prospective study of heart rate variability and mortality in chronic heart failure results of the United Kingdom heart failure evaluation and assessment of risk trial (UK-Heart). Circulation, 98(15), 1510-1516. 